\title{
Microinjection of fluorescent tracers to study neural cell lineages
}

\author{
RICHARD WETTS and SCOTT E. FRASER \\ Department of Physiology and Biophysics, University of California, Irvine and Division of Biology, California Institute of Technology, \\ Pasadena, CA 91125, USA \\ *Present address: Division of Biology, Beckman Institute, 139-74, California Institute of Technology, Pasadena, CA 91125, USA
}

\begin{abstract}
Summary
The examination of cell lineages is an important step towards understanding the developmental events that specify the various cell types in the organism. The mechanisms that control which cell types are formed, their locations, and their numbers remain unknown. Analyses of cell lineage in the frog neural retina have revealed that individual precursors are multipotent and are capable of producing almost any combination of cell types. In addition to giving rise to a wide range of phenotypes, the precursors can give rise to a wide range of clone sizes. Cell lineage studies in other systems indicate that some precursors are multipotent, like those in the retina, while others appear to produce a more
\end{abstract}

restricted range of descendants, perhaps even a single phenotype. These differences in the developmental potential of precursor cells suggest that the nervous system uses several strategies for producing its many cell types. Investigation of these strategies, at the cellular and molecular level, requires more than a description of the normal cell lineages. We are now exploiting the frog neural retina to perform the experimental manipulations needed to elucidate these strategies.

Key words: fate, determination, fluorescent dextran, Xenopus, neural development.

\section{Introduction}

A fundamental issue of developmental biology concerns how different types of cells are created at the right time and in the correct numbers. The proper formation of different cell types requires the specific regulation of many processes during development. These processes include the proliferation of precursor cells, the specification of cell fate, and the terminal differentiation of postmitotic cells. Little is known about the regulation of these key developmental events. An investigation of the mechanisms underlying these events is dependent on an understanding of the cell lineages, the sequence of cell divisions that results in the creation of postmitotic, differentiated cell types. Studies of cell lineages provide information about the numbers of cells and the range of cell types that can be produced by precursor cells at each stage of development. The precursors can be unipotent, giving rise to only one differentiated cell type, while other precursors can be multipotent, forming many different types of cells. A knowledge of the cell lineages and of the potency of the precursors is a necessary first step in understanding the control of cell proliferation and the selection of phenotypes.

The determination of cell lineages requires a means to identify the descendants of single precursor cells. In the nematode $C$. elegans, the small size, accessibility, and transparency of the embryo allowed the lineage of every cell to be determined directly by observation with light microscopy (Kimble and Hirsh, 1979; Sulston and Horvitz, 1977; Sulston et al. 1983). This simple, straightforward approach, however, will not work in vertebrate embryos. First, most vertebrate embryos are relatively inaccessible and opaque, so individual cells cannot be observed directly. Furthermore, vertebrate embryos possess a large number of progenitor cells that are not uniquely identifiable. Thus, unlike $C$. elegans, the same precursor cannot be recognized and followed in different individuals. To overcome these difficulties and to follow lineages in vertebrate embryos, one of the apparently identical precursor cells must be rendered unique so that, later in development, its desendants can be identified and analyzed.

Our approach for rendering a precursor unique is to microinject fluorescently labelled dextrans into single cells. The hydrophilic dextran diffuses throughout the cytoplasm of the injected cell and passes to the daughter cells by cytokinesis (Gimlich and Braun, 1985). The large relative molecular mass of the dextran molecules confines them to the injected cell and its descendants. The dextran is almost inert in animal cells, so it persists for long periods of time, without any toxic effects. The fluorescent moiety allows the labelled cells to be identified in live animals, fixed whole-mounts, or histological sections. Using this approach, our studies 
of the vertebrate nervous system have provided data on the range of cell types produced by neural precursors in several regions and in several species. This paper will focus on our studies of the cell lineages in the neural retina of the frog.

\section{Experimental strategy}

Fluorescent lineage tracers have several advantages over other lineage tracers. (i) The injected cells can be observed in vivo, allowing direct confirmation that only a single cell was injected and permitting observation of cell movements in situ (Kimmel and Warga, 1986; Warga and Kimmel, 1990). (ii) Different fluorescent moieties offer the possibility of labelling neighboring lineages distinctly within individual animals (Sheard and Jacobson, 1987; Wetts and Fraser, 1989). (iii) Labelling cells by microinjection allows the experimenter to control the exact position of the precursor. (iv) The fluorescent tracer can be visualized in essentially every cell type of all species. Unfortunately, as with any injectable tracer, the injection procedure can be difficult, and the tracer can be diluted by mitotic activity. Our experience has been that these potential disadvantages can be avoided easily by appropriate experimental design and by conservative interpretation of the results (Bronner-Fraser and Fraser, 1988, 1989; Fraser et al. 1990; Wetts and Fraser, 1988; Wetts et al. 1989).

The first step in using fluorescent dextran as a lineage tracer is to introduce it into a precursor cell by microinjection. To eliminate movement during the injection, frog embryos are anesthetized with methylsulfonate, placed in a dish with a Sylgard base, and secured with small wire 'staples' placed across their trunk. An epifluorescence microscope, modified so that the stage remains fixed and equipped with a $20 \times$ longworking-distance objective, is used to observe the microinjection procedure. A microelectrode is guided to a cell in the region of interest. The cell is impaled using the capacitance compensation control on the amplifier to 'ring' the microelectrode tip, and penetration is verified by the measurement of a membrane potential. The fluorescent dextran $\left(100 \mathrm{mg} \mathrm{ml}^{-1}\right.$; Molecular Probes) is injected into the cell with pulses of positive current $(4 \mathrm{nA})$. The presence of the fluorescent dextran in a cell can be confirmed immediately after the microinjection using the epifluorescence light of the microscope.

To determine the morphology and location of the injected cell, some animals are fixed immediately after microinjection (Fig. 1A). These data about the precursor cells aids our understanding of the cell lineages observed in other animals, which had received identical injections but had been allowed to develop for several days (e.g. Wetts et al. 1989). Examination of these animals also provides confirmation that our microinjection procedure reliably (more than $90 \%$ of the injections) labels only a single precursor cell. In some of the other cases, the dextran label is seen in a pair of cells
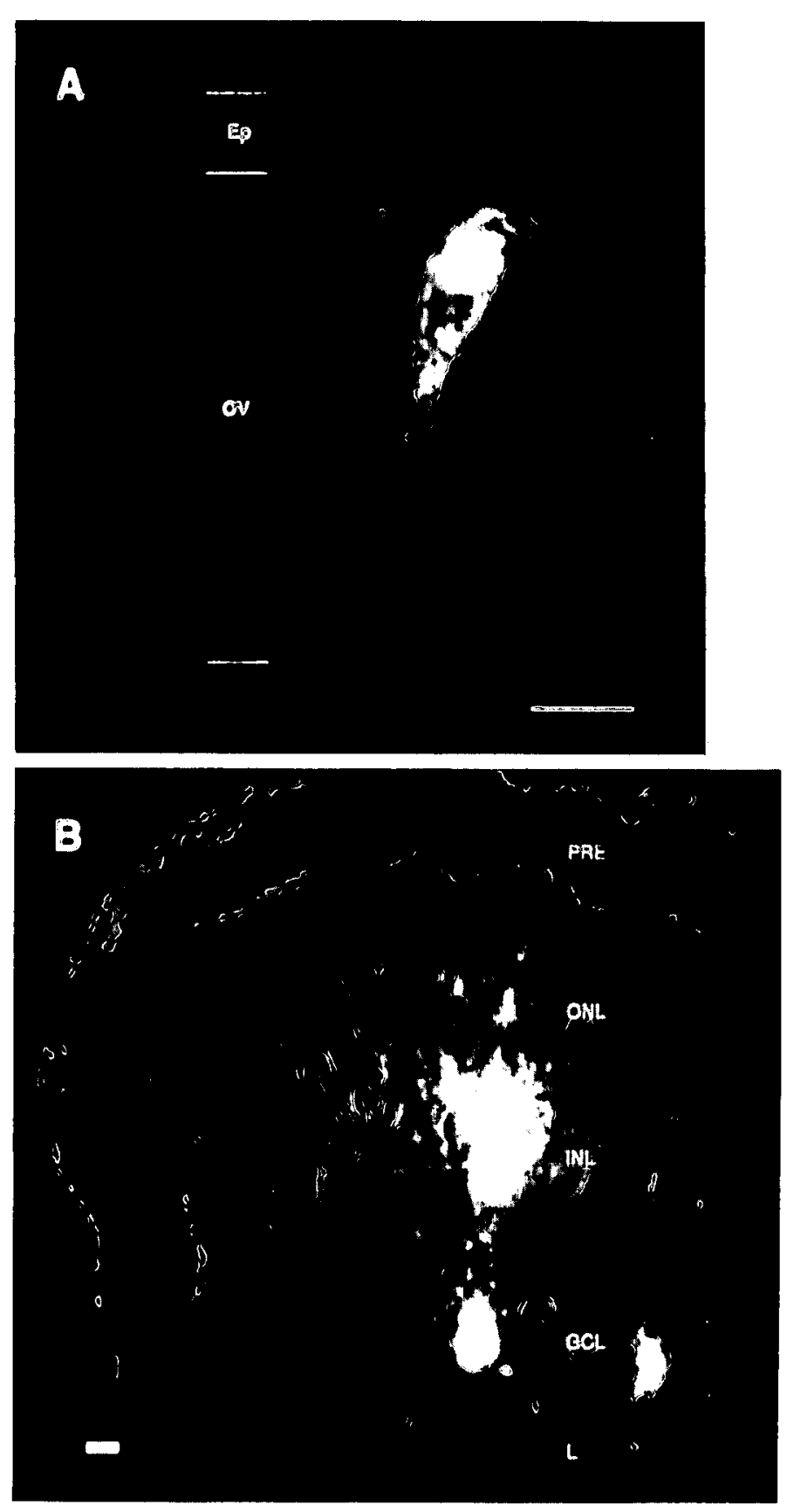

Fig. 1. Rhodamine dextran as a lineage tracer in the frog neural retina. (A) A single precursor cell is filled with rhodamine dextran by microinjection. This cell, labelled at St 23, is located in the optic vesicle (OV); its elongated morphology is characteristic of retinal precursor cells. (B) After several days of development, the descendants of an injected precursor are identified by the rhodamine label. As shown in this example, precursors in the optic vesicle frequently produced clones that spanned all three layers of the neural retina (the ONL, INL, and GCL). Since different cell types are located in each of these layers, the multilaminar distribution of the descendants indicates that their precursor produced multiple cell types. Most of the descendants are aligned in a radial column, indicating that there is little cell mixing during retinal development.

Abbreviations: Ep, epidermis; GCL, ganglion cell layer; INL, inner nuclear layer; L, lens; ONL, outer nuclear layer; OV, optic vesicle; PRE, pigmented retinal epithelium. Scale bars $=20$ microns. 
that were completing cytokinesis. Because descendants of such sister cells would be a clone derived from a single cell (the mother cell), analysis of these descendants provides useful lineage information. In a few cases, two distinct cells are labelled. This can occur when the second cell is labelled inadvertently by dye leakage as the microelectrode is advanced through superficial tissue layers. Because labelling of more than one cell occurs infrequently, it does not effect our conclusions (which are based on numerous cases). Therefore, almost all microinjections label only a single cell, confirming that labelled descendants seen later in development are clonally related cells.

To determine the fate of the injected cell, the animal is given time to develop, then it is fixed and examined either in whole-mount or in histological sections. The descendants of the injected precursor are identified by the fluorescent dextran label. The use of a lightsensitive SIT video camera and video image processing (Imaging Technologies 151 image processor and VIDIM software written by Gary Belford, Jes Stollberg, and Scott Fraser) allow us to distinguish even faintly fluorescent cells and their processes. Visual examination of each clone reveals the locations, the numbers, and the phenotypes of the labelled cells. The range of cell types of a single clone is an indication of the developmental potential of the founder cell. Of course, the observed range is a minimum estimate of the precursor's potential. It is always possible that some cell types are not formed due to chance, or that these cell types are not observed due to cell death or loss of the lineage tracer. The range of cell types that is observed is important data about the normal developmental potential of the precursor cells.

The number of descendants provides an estimate of the position of the precursor in the lineage. For example, a small number of descendants indicates that the labelled precursor was near the end of its lineage. The observed number of descendants indicates the minimum number of divisions that occurred. This minimum number can be calculated by assuming that all of the divisions were 'proliferative' divisions, in which all cells divide until a specific time, then all of them stop dividing simultaneously. 'Stem cell-like' divisions, in which one daughter cell stops dividing while the other continues, produces descendants sequentially; hence more 'stem cell-like' divisions are required to produce the same number of descendants. Furthermore, undercounting the number of descendants due to cell death or loss of the lineage marker also decreases the estimated number of divisions. Because each of these processes causes a decrease in the estimated number of cell divisions, the observed number of descendants provides a minimum value for the number of divisions. This minimum number is a useful indicator of the precursor's position in the lineage.

In addition to the position of the precursor in the lineage, the number of descendants in a clone can provide information about the regulation of proliferation. During development, a precise number of each cell type is produced (Williams and Herrup, 1988), suggesting that proliferation is precisely regulated. However, the relationship between proliferation and the specification of different cell types is poorly understood. It is possible that proliferation and specification are independently regulated during development, but in some cases these events seem to be intertwined. For example, clones of oligodendrocytes form in vitro after all descendants of an O-2A progenitor cell have undergone the same number of divisions, suggesting that proliferation is related to the specification of the precursor cell to form oligodendrocytes (Raff et al. 1988). Thus, analyses of the numbers of descendants extends our understanding of the specification of different cell types.

\section{Cell lineages in the frog eyebud}

The retina is a convenient system for cell lineage studies because its structure is relatively simple, its development is rapid, and its location is accessible for experimental manipulations. The structure of the retina is very similar in all vertebrates, including the frog Xenopus leavis (Adler and Farber, 1986; Dowling, 1987). The outer nuclear layer (ONL) contains the photoreceptors; the inner nuclear layer (INL) contains the interneurons (the horizontal, bipolar, and amacrine cells) and the Muller glial cells; and the ganglion cell layer (GCL) contains the ganglion cells. The small number of cell types and their laminar distribution are advantageous for classifying the descendants of labelled precursors. Developmentally, the retina begins as an evagination of the diencephalon that forms the optic vesicle [St (stage) 20-St 25 in Xenopus]. After contacting the epidermis and inducing the lens, the lateral region of the neuroepithelium invaginates into the cavity of the optic vesicle to form the optic cup (St 26-St 35). The outer layer of cells of the optic cup forms the pigmented retinal epithelium while the inner layer forms the neural retina. In Xenopus, the first postmitotic cells are formed at about St 25 (Beach and Jacobson, 1979; Holt et al. 1988; Jacobson, 1968). By St 37 , mitotic activity in the central part of the eye ceases, and the peripheral edge of the optic cup, called the ciliary margin, becomes the principal proliferative area of the retina (Hollyfield, 1971; Straznicky and Gaze, 1971). The central region of the retina has fully differentiated, forming a functional neural tissue. Thus, in frog, retinal development occurs quickly, finishing within five days of fertilization.

To determine the number and types of cells that could be formed by single retinal precursors, individual cells of the frog eyebud (the optic vesicle at St 22 or the optic cup at St 30) were labelled with rhodamine dextran lineage tracer. Examination of histological sections of the retinas at St 46-52 (2-21 days after labelling) revealed that many precursor cells had formed clones of labelled descendants that were located in all three layers of the retina (Fig. 1B; Table 1). In most of the clones, the labelled cells were distributed in a radial column, with little spread circumferentially 
Table 1. Laminar distribution of labelled cells descended from precursors in the optic cup (St 30)

\begin{tabular}{lcccc}
\hline $\begin{array}{l}\text { Laminar } \\
\text { distribution } \\
\text { of descendants }\end{array}$ & $\begin{array}{c}\text { Number of } \\
\text { descendants } \\
\text { per clone* }\end{array}$ & Probability† & $\begin{array}{c}\text { Expected } \\
\text { number } \\
\text { of clones }\end{array}$ & $\begin{array}{c}\text { Observed } \\
\text { number } \\
\text { of clones }\end{array}$ \\
\hline ONL only & $2.3 \pm 0.9$ & 0.06 & 2 & 3 \\
INL only & $4.4 \pm 0.9$ & 0.12 & 4 & 8 \\
GCL only & $1.0 \pm 0.0$ & 0.17 & 6 & 2 \\
ONL and INL & $6.0 \pm 0.6$ & 0.29 & 9 & 6 \\
ONL and GCL & $2.0 \pm 0.0$ & 0.08 & 3 & 2 \\
INL and GCL & $6.0 \pm 0.8$ & 0.15 & 5 & 6 \\
All 3 layers & $10.7 \pm 1.0$ & 0.83 & 27 & 29
\end{tabular}

This analysis of the laminar distribution of 56 clones derived from optic cup precursors shows that most are spread over more than one layer. Because each layer is composed of characteristic cell types different from the cell types in the other layers, the multilaminar distribution indicates that these clones are multipotent. Thirteen clones have all of their descendants in one layer; some or all of these clones must have produced only a single cell type. For each type of laminar distribution (i.e., for each row), the mean number of descendants (second column) was used to calculate the probability that this number of cells would have the observed laminar distribution by chance alone (third column). Because of the small size of these unilaminar clones, the probability that all of the descendants came to be located in the same layer by chance alone is reasonably high. Further, these probabilities were used to calculate the expected numbers of clones for each kind of laminar distribution (fourth column); the expected numbers are similar to the actual numbers of clones (fifth column; $P=0.60$ by chi-square test). Thus, there is no evidence from this analysis that any of the precursor cells in the optic cup were committed to producing a specific cell type.

Abbreviations: GCL, ganglion cell layer; INL, inner nuclear layer; ONL, outer nuclear layer. *Mean \pm S.E.M. †The probability that a single descendant will be located in a specific layer is calculated from the ratio of all 444 labelled descendants in the three layers: $17 \%$ were located in the GCL, $59 \%$ in the INL, and $24 \%$ in the ONL. (This ratio is similar, $P=0.39$ by chi-square test, to the ratio of cells in the different laminae of unlabelled, normal eyes: $17 \%$, $55 \%$, and $28 \%$, respectively). These percentages were used to calculate the probability, for each row, that given the observed mean number of descendants, the clone will have that laminar distribution. The probabilities do not sum to $100 \%$ because they are calculated independently for each row.

within the plane of the retina. For each clone, the number of labelled cells visible in each section was determined. After microinjection of an optic vesicle cell (St 22), the number of descendants ranged from 1-42, with a mean of 14.3 (s.E.M. $=1.3 ; n=62$ clones). When the precursor cell was labelled later in development, there were fewer descendants. Optic cup cells (St 30) produced clones of $1-35$ cells, with a mean of 10.8 (S.E.M. $=1.2 ; n=56$ clones). Since some retinal cells withdraw from the mitotic cycle between St 22 and St 30 (Holt et al. 1988), it is not surprising that we observed smaller clones after labelling precursors at the later developmental stage. At both St 22 and St 30, the precursors give rise to clone sizes that can be any value in a relatively large range (Fig. 2). That is, after labelling precursors at a specific developmental stage, cell proliferation apparently does not stop at a specific time or after a specific number of divisions. One explanation for this apparent lack of specificity is that each cell's decision to stop dividing may be partly a


Fig. 2. Histograms of the frequency distributions of clone sizes for precursors labelled at the optic vesicle (St 22) and the optic cup (St 30) stages. Precursors labelled at St 30 (top histogram) produce more small clones and fewer large clones than do precursors labelled at St 22 (bottom histogram). This difference between St 30 and St 22 is not surprising, because precursors at St 30 have less time to proliferate before the end of the birthdate period (at St 37). Precursors at both stages produce a relatively wide range of clone sizes; this wide range suggests that the regulation of proliferation might involve a stochastic process.

matter of chance (Turner et al. 1990; Wetts and Fraser, 1988).

In some descendants, the fluorescent dextran fills the cells' processes, and specific types of cells can be recognized by their characteristic morphology (Dowling, 1976; Ramon y Cajal, 1972). For both optic vesicle cells and optic cup cells, all major cell types were present in clones that included other identified cell types (Wetts and Fraser, 1988 and unpublished observations). Many clones included both neurons and Muller glial cells. In some of the smallest clones, each of the descendants was clearly different from the others. For example, one clone was composed of a ganglion cell, a photoreceptor and an unidentified cell of the INL. Because at least some of the eyebud cells are multipotent as late as their last cell division, it is likely that the interactions and molecular events that specify cell types occur late in the development of the frog retina.

The multipotency of retinal precursors and the variety of the numbers of descendants have been observed independently in several species using a variety of techniques. Using horseradish peroxidase as 
the lineage tracer, Holt et al. (1988) observed clones of multiple cell types in the frog retina similar to the clones described above. Retroviral lineage tracers have revealed multipotent precursors in the retina of both rodents and aves (Fekete et al. 1990; Turner and Cepko, 1987; Turner et al. 1990). In all of these experiments, even the smallest clones sometimes were composed of multiple cell types, suggesting that specification of retinal cell types occurs at or after the last division (Turner and Cepko, 1987; Wetts and Fraser, 1988). Thus, the time at which cell types are specified seems to be similar in the vertebrate retina and the Drosophila retina (Lawrence and Green, 1979; Ready et al. 1976). The multipotency of retinal precursors further suggests that cell-cell interactions are important in the specification of the retinal cell types. Evidence consistent with such interactions has been obtained in both flies (Banerjee and Zipursky, 1990; Tomlinson, 1988) and frogs (Reh, 1987; Reh and Tully, 1986).

From the data reviewed above, we have shown that retinal precursors can give rise to any of the cell types in the neural retina. This is, in fact, a minimum estimate of the potency of these precursors, because it is not known whether the retinal precursors could give rise to nonretinal cell types. If a retinal cell was moved to another location, it might be able to produce additional cell types. Alternatively, it is possible that the retinal precursors are committed to producing only retinal cell types and are unable to produce other types of neurons or glia. This type of regional commitment has been observed in the spinal cord. Spinal cord precursors, which are now known to be multipotent (Hartenstein, 1989; Leber et al. 1990; Stern et al. 1988), can differentiate into retinal cell types if they are transplanted into the prospective eye region at early neural plate stages (Szaro et al. 1985). Thus, early in development, the potential of spinal cord precursors appears to be greater than their actual fate. Later in development, the spinal cord cells do not form retinal derivatives, suggesting that they become committed to forming a specific region of the nervous system. Yet these cells remain multipotent, so the specification of the final cell type occurs still later in development. The identification and study of these sequential restriction events will provide important insights into the mechanisms that form the different cell types of the nervous system.

\section{Cell lineages in the ciliary margin}

During embryogenesis in Xenopus, the cells in the central region of the optic cup undergo their last divisions between St 25 and 37 (Holt et al. 1988; Jacobson, 1968; Straznicky and Gaze, 1971). Those cells at the periphery form the ciliary margin, a group of proliferating cells in lower vertebrates that adds new cells at the edge of the retina throughout life. The ciliary margin is a steady-state system in which all developmental events are occurring simultaneously. That is, at all stages after St 37, the cells at the edge of the retina are dividing, those located more centrally are becoming postmitotic, the next cells are differentiating, and the most centrally located cells are differentiated and functioning. The characteristics of the ciliary margin provide unique opportunities to study the regulation of proliferation and differentiation in the frog retina.

To determine the lineages of ciliary margin cells, individual cells were microinjected with rhodamine dextran at St 39-41, when only the ciliary margin cells are proliferating in the retina (Wetts et al. 1989). In animals fixed 2-21 days after microinjection, the labelled descendants formed radial columns that extended over all layers of the retina. Like the optic vesicle cells, some ciliary margin cells produced all of the major cell types of the neural retina, including the Muller glial cell. Even the smallest clones were composed of multiple cell types. This multipotency of the ciliary margin cells is similar to the multipotency of the optic vesicle and optic cup cells. In contrast to the eyebud clones, some of the ciliary margin clones had descendants both in the neural retina and in the pigmented retina. Although no pigmented descendants were observed in the eyebud clones, this cannot be taken as evidence that the cells are incapable of making pigment cells. It is possible that optic vesicle precursors have the potential to produce pigment cells, but fail to do so because of the spatial segregation of the envionmental cues, either permissive or instructive, that specify the neural and pigment phenotypes. The similarities and differences between the ciliary margin and eyebud cells provide an experimental tool for determining whether these precursors use similar or different developmental mechanisms for specifying cell fate in the retina.

Quantitative analysis revealed some differences in the pattern (and hence regulation) of proliferation in the ciliary margin compared to the eyebud. The number of descendants per ciliary margin clone was highly variable (1-104 cells), but the values appeared to be clustered into two groups (Wetts et al. 1989). Approximately two-thirds of the clones were small in size, with less than 37 cells, while all of the remaining clones were large, with 56 or more cells. Since the ciliary margin produces new cells throughout life, at least some of the margin cells must be self-renewing stem cells. The large clones could have resulted from microinjecting stem cells that can undergo an unlimited number of divisions. Such stem cells would be expected to form very large clones (as many as thousands of cells over the life of the animal). An inherent feature of using rhodamine dextran as the lineage tracer is that it becomes diluted with each division. The dye intensity in many of our large clones fits the pattern expected for stem cell division. The first descendants of a stem cell to become postmitotic, located near the central retina (Straznicky and Gaze, 1971), should be brightest as they inherited the dye before any significant dilution of the dextran label had occurred. In our large clones, the most central descendants were labelled brightly. Because a stem cell continues to divide and dilute the dextran, its later 
descendants should be faintly labelled; cells located more peripherally were fainter than the central descendants in the same clone. Cells adjacent to the ciliary margin were frequently unlabelled, as expected for cells that were last-born, after the dye concentration had decreased to undetectable levels. Thus, the visibly labelled cells in our largest clones probably represent the early descendants produced from a stem cell precursor.

Our lineage data suggest there may be two types of precursors in the ciliary margin: stem cells that can undergo an unlimited number of divisions (which produced our large clones) and non-stem cells that undergo only a limited number of divisions (which produced our small clones). However, our data cannot be taken as proof of two inherently distinct precursors. For example, it is possible that position within the ciliary margin is the major determinant of the proliferative pattern of any one precursor. In this scenario, those cells at the peripheral edge of the ciliary margin are the stem cells. As they divide, some of the daughters remain at the peripheral edge, and these remain stem cells. Other daughters become displaced away from the edge; these then undergo several divisions before they become postmitotic. Thus, the functional differences between the stem and non-stem cells might arise from their differences in position and not from any trait intrinsic to the cells themselves.

\section{Studies of cell lineage in other areas of the nervous system}

Although this paper has concentrated on the use of injectable cell lineage tracers in the amphibian visual system, the approach is applicable to a variety of tissues and species. Unlike retrovirus lineage tracing experiments, in which the virus is limited as to which species it can infect, there is no component of the microinjection technique that is species specific. The requirements are very simple and general: some means to stabilize the embryo, a path for the microelectrode to pass, and a stable impalement of the cell. In the case of the avian embryo, the experiment is accomplished simply, by windowing the egg and placing it on the stage of an epifluorescence microscope. Even cells deep within the embryo can be impaled. For example, cells in the basal plate and floor plate of the developing neural tube can be accessed by bringing the electrode through the roof plate and impaling the cells from their lumenal surfaces. Cells in the tissues through which the electrode passes are not labelled by the dextran because no current is applied to expel the dye. Artifactual labelling due to dye leakage and/or cell injury can be directly assessed by viewing the specimen with the epifluorescence microscope immediately after the microelectrode is withdrawn

The microinjection technique has been used to study cells in the basal plate, alar plate and floor plate of the developing avian neural tube, ranging from the rhombencephalon (Fraser et al. 1990) to the spinal cord
(Stern et al. 1988). In the spinal cord, the descendants of the injected cell disperse considerably, spreading rostrocaudally as much as a somite length. There appeared to be no fixed boundaries to this spreading; those few restrictions that have been discerned appear to be a direct result of physical interactions with the somites (Stern et al. 1991). The phenotypes within single spinal cord clones were diverse (Stern et al. 1988), in agreement with studies using retroviral tracers (Leber et al. 1990). This diversity suggests that cell phenotype is assigned late in the cell lineage, as described above for the neural retina.

In contrast to other axial levels, the descendants of single precursors in the rhombencephalon differentiated into only one or two cell types (A. Lumsden, S. Fraser and R. Keynes, unpublished data). In many cases, all of the 8 to 32 cells in a clone were of identical phenotypes, even though they were intermixed with unlabelled descendants of neighboring cells that were developing into other cell types. Although the rhombencephalon clones dispersed widely, they appeared to be restricted by the boundaries of the rhombomeres (hindbrain segments; see Keynes and Lumsden, 1990). The descendants of most of the precursors labelled before the appearance of the rhombomeres were confined to one rhombomere; those from injections performed after the boundaries became visible were always restricted (Fraser et al. 1990). These results strongly suggest that cell phenotype and segmental identity is imposed on the cells of the developing hindbrain many divisions before the cells become postmitotic. This apparent early commitment contrasts with other areas of the nervous system, such as the neural retina (Fekete et al. 1990; Holt et al. 1988; Turner and Cepko, 1987; Turner et al. 1990; Wetts and Fraser, 1988; Wetts et al. 1989), the spinal cord (Hartenstein, 1989; Leber et al. 1990; Stern et al. 1988), and the neural crest (Bronner-Fraser and Fraser, 1988, 1989). It requires future work to establish which of these developmental strategies is most common. For example, retroviral studies of the cortex have provided evidence suggesting both that the precursors are restricted in potential (Barfield et al. 1990; Luskin et al. 1988; Parnavelas et al. 1990) and that they are multipotent (Price and Thurlow, 1988; Walsh and Cepko, 1988); microinjection studies of the diencephalon demonstrate a restriction to cell mixing at neuromere boundaries (M. Figdor and C. Stern, personal communication). Such regional differences can be exploited in a comparative analysis to gain insights into the mechanisms involved in the specification of cell phenotype and position. Because the microinjection approach is not species or tissue specific, it is well suited to such a strategy.

\section{Concluding remarks}

Cell lineages observed in normal animals provide useful information about what cell types share common ancestors and when during the lineage the common ancestor occurs. However, this straightforward analysis 
must be viewed as a means rather than an end; lineages in normal animals cannot indicate what causes them to diverge or why the precursors become restricted to a specific fate. For example, a unipotent clone (i.e. all of the descendants have differentiated into the same cell type) need not be the product of a fixed cell lineage. Instead, an uncommitted precursor could have produced descendants that all differentiated into the same cell type due to chance or due to physical barriers that kept the descendants restricted to a sub-region, thereby exposing all of them to the same extrinsic determinants. Similarly, the finding of a multipotent clone does not indicate the absence of strict lineage control; a precursor could be committed to form a specific set of cells composed of multiple cell types. To assay whether a precursor cell is committed to producing a specific cell type(s), experimental manipulations must be performed that challenge the precursor to produce additional cell types. Clearly, the normal cell lineages must be elucidated before one can determine whether the normal fate has changed in response to such a manipulation.

Our studies of the cell lineages in the normal frog retina are but the first steps in dissecting the developmental events leading to the formation of different cell types and their numbers. Our experiments revealed that precursors in the retina give rise to multiple cell types and to a variety of clone sizes. These results now raise new questions not only about the mechanisms that regulate cell proliferation and the events that specify different cell types, but also about the molecular bases of these developmental events. Our experimental strategy provides a means for obtaining answers to these questions; data from the normal cell lineages are needed to design, execute, and interpret the experiments in which the commitment of the precursor cells are tested by experimental manipulations. The frog retina is one of the systems in which a variety of such manipulations can be performed. This approach, which combines experimental perturbations with cell lineage analysis, should help to elucidate the mechanisms that regulate proliferation and that specify cell phenotype.

This work was supported by grants from the NIH (EY08153, EY08363, and HD25390), the NSF (BNS 8608356), and the Monsanto Corp. We thank M.S. Carhart, S. Burgan, and $T$. Joe for their technical assistance.

\section{References}

Adler, R. and Farber, D. (eds) (1986). The Retina: A Model for Cell Biology Studies, Part I and II. Academic Press, Orlando.

BanerJeE, U. and ZIPURSKY, S. L. (1990). The role of cell-cell interactions in the development of the Drosophila visual system. Neuron 4, 177-187.

Barfield, J. A., Parnavelas, J. G. and Luskin, M. B. (1990). Separate progenitor cells give rise to neurons, astrocytes, and oligodendrocytes in the rat cerebral cortex. Soc. Neurosci. Abstr. 16, 1272.

BeACH, D. H. AND JacoBson, M. (1979). Patterns of cell proliferation in the retina of the clawed frog during development. J. comp. Neurol. 183, 603-614.

Bronner-Fraser, M. and Fraser, S. E. (1988). Cell lineage analysis reveals multipotency of some avian neural crest cells. Nature 335, 161-164.

Bronner-Fraser, M. and Fraser, S. E. (1989). Developmental potential of avian trunk neural crest cells in situ. Neuron 3, $755-766$.

Dowling, J. E. (1976). Physiology and morphology of the retina. In Frog Neurobiology (R. Llinas and W Precht, eds). Springer Verlag, NY. pp. 278-296.

Dowling, J. E. (1987). The Retina. An Approachable Part of the Brain. Belknap Press, Cambridge, Mass.

Fekete, D. M., Ryder, E. F., Stoker, A. W. and Cepko, C. L. (1990). Neuronal lineage and determination in the chick retina using retroviruses and cell transplants. Soc. Neurosci. Abstr. 16, 1272.

Fraser, S. E., Keynes, R. and Lumsden, A. (1990).

Segmentation in the chick embryo hindbrain is defined by cell lineage restrictions. Nature 344, 431-435.

GimLich, R. L. AND BRAUN, J. (1985). Improved fluorescent compounds for tracing cell lineage. Devl Biol. 109, 509-514.

HaRTENSTEIN, V. (1989). Early neurogenesis in Xenopus: The spatio-temporal pattern of proliferation and cell lineages in the embryonic spinal cord. Neuron 3, 399-411.

Hollyfield, J. G. (1971). Differential growth of the neural retina in Xenopus laevis larvae. Devl Biol. 24, 264-286.

Holt, C. E., Bertsch, T. W.; Ellis, H. M. and Harris, W. A. (1988). Cellular determination in the Xenopus retina is independent of lineage and birth date. Neuron 1, 15-26.

JACOBSON, M. (1968). Cessation of DNA synthesis in retinal ganglion cells correlated with the time of specification of their central connections. Devl Biol. 17, 219-232.

Keynes, R. AND LumSDEN, A. (1990). Segmentation and the origin of regional diversity in the vertebrate central nervous system. Neuron 2, 1-9.

Kimble, J. E. And Hirsh, D. (1979). Postembryonic cell lineages of the hermaphrodite and male gonads in Caenorhabditis elegans. Devl Biol. 70, 396-417.

Kimmel, C. B. AND WarGa, R. M. (1986). Tissue-specific cell lineages originate in the gastrula of the zebrafish. Science 231, $365-368$.

Lawrence, P. A. and Green, S. M. (1979). Cell lineage in the developing retina of Drosophila. Devl Biol. 71, 142-152.

Leber, S. M., Breedlove, S. M. and Sanes, J. R. (1990). Lineage, arrangement, and death of clonally related motoneurons in chick spinal cord. J. Neurosci 10, 2451-2462.

Luskin, M. B., Pearlman, A. L. and Sanes, J. R. (1988). Cell lineage in the cerebral cortex of the mouse studied in vivo and in vitro with a recombinant retrovirus. Neuron 1, 635-647.

Parnavelas, J. G., Barfield, J. A. And Luskin, M. B. (1990). Lineage relationships of pyramidal and nonpyramidal neurons in the rat cerebral cortex. Soc. Neurosci. Abstr. 16, 1272.

Price, J. and Thurlow, L. (1988). Cell lineage in the rat cerebral cortex: a study using retroviral-mediated gene transfer. Development 104, 473-482.

RafF, M. C., Lillien, L. E., Richardson, W. D., Burne, J. F. AND Noble, M. (1988). Platelet-derived growth factor from astrocytes drives the clock that times oligodendrocyte development in culture. Nature 333, 562-565.

Ramon y Cajal, S. (1972). The Structure of the Retina. (Compiled and transl. by S.A. Thorpe and M. Glickstein). Charles C. Thomas, Springfield, IL.

Ready, D. F., Hanson, T. E. and Benzer, S. (1976). Development of the Drosophila retina, a neurocrystalline lattice. Devl Biol. 53, 2.17-240.

ReH, T. A. (1987). Cell-specific regulation of neuronal production in the larval frog retina. $J$. Neurosci. 7, 3317-3324.

ReH, T. A. AND TUlLy, T. (1986). Regulation of tyrosine hydroxylase-containing amacrine cell number in larval frog retina. Devl Biol. 114, 463-469.

ShEARd, P. AND Jacobson, M. (1987). Clonal restriction boundaries in Xenopus embryos shown with two intracellular lineage tracers. Science 236, 851-854.

Stern, C. D., Fraser, S. E., Keynes, R. J. and Primmett, D. R. (1988). A cell lineage analysis of segmentation in the chick embryo. Development 104 Suppl, 231-244. 
Stern, C. D., Jaques, K. F., Lim, T.-M., Fraser, S. E. and KeYNES, R. J. (1991). Segmental lineage restrictions in the chick embryo spinal cord depend on the adjacent somites. Development 113, 239-244.

StRaZnicky, K. AND GAZE, R. M. (1971). The growth of the retina in Xenopus laevis: An autoradiographic study. J. Embryol. exp. Morph. 26, 67-79.

Sulston, J. E. AND HoRvitz, H. R. (1977). Post-embryonic cell lineages of the nematode Caenorhabditis elegans. Devl Biol. 56, 110-156.

Sulston, J. E., Schierenberg, E., White, J. G. and Thomas, J. $\mathrm{N}$. (1983). The embryonic cell lineage of the nematode Caenorhabditis elegans. Devl Biol. 100, 64-119.

Szaro, B., Ide, C., Kaye, C. and Tompkins, R. (1985). Regulation in the neural plate of Xenopus laevis demonstrated by genetic markers. J. exp. Zool. 234, 117-129.

Tomlinson, A. (1988). Cellular interactions in the developing Drosophila eye. Development 104, 183-193.

Turner, D. L. ANd Cepko, C. L. (1987). A common progenitor for neurons and glia persists in rat retina late in development. Nature 328, 131-136.

Turner, D. L., Snyder, E. Y. and Cepko, C. L. (1990). Lineageindependent determination of cell type in the embryonic mouse retina. Neuron 4, 833-845.

Walsh, C. AND Cepko, C. L. (1988), Clonally related cortical cells show several migration patterns. Science 241, 1342-1345.

Warga, R. M. and Kimmel, C. B. (1990). Cell movements during epiboly and gastrulation in zebrafish. Development 108, 569-580.

WeTtS, R. AND Fraser, S. E. (1988). Multipotent precursors can give rise to all major cell types of the frog retina. Science 239, $1142-1145$.

WeTtS, R. AND FRASER, S. E. (1989). Slow intermixing of cells during Xenopus embryogenesis contributes to the consistency of the blastomere fate map. Development 105, 9-15.

Wetts, R., Serbedzija, G. N. and Fraser, S. E. (1989). Cell lineage analysis reveals multipotent precursors in the ciliary margin of the frog retina. Devl Biol. 136, 254-263.

Williams, R. W. and Herrup, K. (1988). The control of neuron number. A. Rev. Neurosci. 11, 423-453. 